\title{
Pegaspargase: A Review in Acute Lymphoblastic Leukaemia
}

\author{
Young-A. Heo ${ }^{1}$. Yahiya Y. Syed ${ }^{1}$. Susan J. Keam ${ }^{1}$
}

Published online: 27 April 2019

(c) Springer Nature 2019, corrected publication 2019

\begin{abstract}
Pegaspargase $\left(\mathrm{Oncaspar}^{\circledR}\right)$, a pegylated form of native Escherichia coli-derived L-asparaginase (hereafter referred as E. coli L-asparaginase), is indicated in the USA and EU for the treatment of acute lymphoblastic leukaemia (ALL) as a component of multi-agent chemotherapy in paediatric and adult patients. Relative to E. coli L-asparaginase, pegaspargase has a prolonged circulation time, thereby offering less frequent administration. Moreover, pegylation of $E$. coli L-asparaginase may diminish the immunogenicity of the enzyme. Based on extensive evidence, intramuscular (IM) or intravenous (IV) administration of pegaspargase as a component of a multi-agent chemotherapy is an effective first-line treatment for paediatric and adult patients with ALL, as well as for the treatment of paediatric and adult patients with ALL and hypersensitivity to $E$. coli L-asparaginase. Pegaspargase had a manageable tolerability profile in paediatric and adult patients with newly diagnosed ALL, with the most commonly occurring adverse events being generally consistent to that seen with $E$. coli L-asparaginase. Pegaspargase treatment in patients with relapsed ALL and hypersensitivity to $E$. coli L-asparaginase had a similar tolerability profile to that observed in patients with newly diagnosed ALL. Given the potentially reduced immunogenicity and more convenient dosage regimen over $E$. coli $\mathrm{L}$-asparaginase, pegaspargase remains an important and effective treatment option for paediatric and adult patients with ALL, including those with hypersensitivity to $E$. coli L-asparaginase.
\end{abstract}

\section{Pegaspargase: clinical considerations in ALL}

Pegylation of E. coli L-asparaginase prolongs elimination half-life (permitting biweekly therapy) and potentially reduces immunogenicity

Similar to native $E$. coli $\mathrm{L}$-asparaginase in paediatric and adult patients with ALL with respect to asparagine depletion and survival outcomes

Manageable tolerability profile; no new safety signals

The manuscript was reviewed by: C. Cerchione, Hematology Unit, Istituto Scientifco Romagnolo per lo Studio e la Cura dei Tumori (IRST) IRCCS, Meldola, Italy; D.J. DeAngelo, DanaFarber Cancer Institute and Harvard University, Boston, MA, USA; J.M. Ribera, ICO Badalona-Hospital Germans Trias i Pujol, Universitat Autònoma de Barcelona, Badalona, Spain.

Electronic supplementary material The online version of this article (https://doi.org/10.1007/s40265-019-01120-1) contains supplementary material, which is available to authorized users.

Additional information for this Adis Drug Evaluation can be found at https://doi.org/10.6084/m9.figshare.8001764.

Young-A. Heo

demail@ springer.com

1 Springer, Private Bag 65901, Mairangi Bay, Auckland 0754, New Zealand

\section{Introduction}

Acute lymphoblastic leukaemia (ALL) is a heterogeneous haematological malignancy that occurs mainly in children (median age at diagnosis $\approx 15$ years); however, ALL in adults accounts for $\approx 20 \%$ of all leukaemia cases [1]. ALL is typically characterized by the proliferation of large number of immature lymphoid cells in the bone marrow, peripheral blood and other organs. With an improved understanding of the pathogenesis of ALL and the recent advances in targeted therapies, the rates of complete remission (CR) and overall survival (OS) in patients with ALL have improved significantly, primarily in the paediatric population. Indeed, 5-year OS in children with ALL is $86-89 \%$, with the rate declining with increased age (OS in adults $\approx 41 \%$ [2]) [1].

The treatment of ALL includes long-term use of multiagent chemotherapy $[1,2]$, of which asparaginase is a cornerstone component $[2,3]$. Asparagine is a nonessential amino acid for normal cell growth but is considered essential for leukaemic cells as these cells are dependent on exogenous sources of asparagine for survival [3]. Asparaginase selectively kills leukaemic cells by depleting asparagine levels [3]. Clinical experience with asparaginase therapy as a component of multi-agent chemotherapy showed improved clinical outcomes in patients with ALL [4, 5].

Native Escherichia coli-derived L-asparaginase (hereafter referred as E. coli $\mathrm{L}$-asparaginase) is one of the asparaginase preparations that have been used for the treatment of ALL 
since 1970s; however, its use is associated with high immunogenicity, which can be manifested by the occurrence of hypersensitivity reactions [5] and/or the neutralization of asparaginase activity without any signs of hypersensitivity (i.e. silent inactivation) [6]. Moreover, E. coli L-asparaginase has to be administered frequently (i.e. three times weekly), as the drug has a short elimination half-life [3]. Consequent to these limitations, pegylated formulations of E. coli $\mathrm{L}$-asparaginase have been developed, including pegaspargase $\left(\right.$ Oncaspar $\left.^{\circledR}\right)$ [with a succinimidyl succinate linker] and calaspargase pegol (with a succinimidyl carbamate linker). Pegylation of E. coli L-asparaginase extends circulation time of the enzyme and diminishes immunogenicity [7]. The favourable pharmacokinetic and immunogenicity profile of pegylated formulations over $E$. coli L-asparaginase helps to reduce the occurrence of hypersensitivity reactions, the development of neutralizing antibodies and the administration frequency, while maintaining its anti-leukaemic efficacy.

Pegaspargase $\left(\mathrm{Oncaspar}^{\circledR}\right)$ solution for injection or infusion is approved in the USA [8] and EU [9] as a component of multi-agent chemotherapy for the treatment of ALL in paediatric and adult patients (Sect. 5). This review considers the pharmacological properties, therapeutic efficacy and tolerability data relevant to the use of the approved dosage of pegaspargase solution for the treatment of ALL in paediatric and adult patients, including those with hypersensitivity to E. coli L-asparaginase. Discussion of calaspargase pegol (recently approved in the USA [10]) is outside the scope of this review.

\section{Pharmacological Properties of Pegaspargase}

\subsection{Pharmacodynamic Properties}

Pegaspargase is formulated by conjugating E. coli L-asparaginase with polyethylene glycol $[3,8,9]$. Unlike normal cells, leukaemic cells have low expression levels of asparagine synthetase [3] and are normally dependent on serum asparagine for survival. Pegaspargase hydrolyses asparagine into aspartic acid and ammonia, depleting asparagine levels and inhibiting protein, DNA- and RNA-syntheses, resulting in leukaemic cell death $[3,8,9]$. Asparaginase activity of $>0.1 \mathrm{IU} / \mathrm{mL}$ is generally the target concentration considered adequate for asparagine depletion [11, 12]. The antileukaemic effect of L-asparaginase (and therefore pegaspargase) is due to prolonged $\mathrm{L}$-asparagine depletion in blood and cerebrospinal fluid (CSF) $[8,9]$.

In animal studies, single, weekly or biweekly intraperitoneal injections of pegaspargase cured most mice infected with lymphoma or lymphosarcoma cells and induced complete response or partial response in most dogs bearing spontaneous lymphoma or lymphosarcomas [13]. In addition, in patients with newly diagnosed ALL, the immediate death of leukameic cells in vivo (measured by rhodamine fluorescence) following pegaspargase $2500 \mathrm{U} / \mathrm{m}^{2}$ was the same as that following E. coli L-asparaginase or Erwiniaderived asparaginase $25,000 \mathrm{U} / \mathrm{m}^{2}$ [9].

\subsection{Pharmacokinetic Properties}

In a population pharmacokinetic analysis in paediatric patients with ALL $(n=1342)$, the pharmacokinetic profile of multiple doses of IV pegaspargase $2500 \mathrm{IU} / \mathrm{m}^{2}$ was best described by a multiple compartment transit model, with increased clearance in the last compartment of transit chain [14].

Pharmacokinetic analysis of pegaspargase was based on an enzymatic assay measuring asparaginase activity following intramuscular (IM) and intravenous (IV) administration of $2500 \mathrm{IU} / \mathrm{m}^{2}$ in patients with ALL $(n=45-52)$ $[8,9]$. Following a single IM injection of pegaspargase, the peak asparaginase activity $\left(\mathrm{C}_{\max }\right)$ of $\approx 1 \mathrm{IU} / \mathrm{mL}$ was reached by day 5 ; the mean absorption half-life was 1.7 days. Following a single IV infusion of pegaspargase during the induction phase, a $\mathrm{C}_{\max }$ of $1.6 \mathrm{IU} / \mathrm{mL}$ was reached in $1.25 \mathrm{~h}$; the area under the asparaginase activity time curve (AUC) from time zero to infinity $\left(\mathrm{AUC}_{\text {inf }}\right)$ was $16.6 \mathrm{IU} / \mathrm{mL} /$ day $[8,9]$. Pegaspargase had a relative bioavailability of $82 \%$ after the first IM dose and $98 \%$ after repeated dosing [8].

After single and repeated doses of pegaspargase $2500 \mathrm{IU} / \mathrm{m}^{2}$, clinically relevant asparaginase activity was maintained over the entire dosing interval of two weeks [9]. In the DFCI 11-001 trial (Sect. 3.1.2), following a single dose of IV pegaspargase $2500 \mathrm{IU} / \mathrm{m}^{2}$ during the induction phase, $94 \%$ of ALL patients sustained asparaginase activity $\geq 0.1 \mathrm{IU} / \mathrm{mL}$ for 18 days. Furthermore, following biweekly dosing of IV pegaspargase during the post-induction phase, a nadir asparaginase activity of $>0.4 \mathrm{IU} / \mathrm{mL}$ was seen in all patients from weeks $7-25$ [9].

In ALL patients, the mean volume of distribution at steady state was estimated to be $1.86 \mathrm{~L} / \mathrm{m}^{2}$ after a single IM injection of pegaspargase and $1.95 \mathrm{~L}$ following a single IV infusion, based on non-compartmental analysis [8, 9]. Pegaspargase is expected to be metabolised by proteolytic enzymes distributed in the tissues [9]. The estimated mean elimination half-life of pegaspargase following a single IM and IV dose was 5.5 and 5.3 days, respectively; the estimated clearance with a single IM and IV dose was 
$0.17 \mathrm{~L} / \mathrm{m}^{2} /$ day and $0.2 \mathrm{~L} /$ day. A population pharmacokinetic analysis based on data from the AALL0704, DFCI 11-001 and CCG-1962 studies (Sect. 3) indicated that the volume of distribution increased slightly more than proportional to body surface area (BSA) and the clearance increased approximately proportional to BSA [9].

Pegaspargase had a prolonged elimination half-life relative to other asparaginase preparations [9]. Following a single IM injection of pegaspargase $2500 \mathrm{IU} / \mathrm{m}^{2}$, E. coli $\mathrm{L}$-asparaginase $25,000 \mathrm{IU} / \mathrm{m}^{2}$ or Erwinia-derived asparaginase $25,000 \mathrm{IU} / \mathrm{m}^{2}$ in paediatric patients with newly diagnosed ALL, the elimination half-life of pegaspargase was substantially longer than seen with $E$. coli L-asparaginase and Erwinia-derived asparaginase (5.7 vs. 1.3 and 0.65 days) [9].

In patients with relapsed ALL and hypersensitivity to $E$. coli $\mathrm{L}$-asparaginase, the mean elimination half-life following IM administration of pegaspargase $2500 \mathrm{IU} / \mathrm{m}^{2}$ on day 1 and 15 during the induction was 2.7 days, which was shorter than in nonhypersensitive patients with relapsed ALL (8 days) [9]. The AUC in hypersensitive and nonhypersensitive patients with relapsed ALL was 3.52 and $10.35 \mathrm{IU} / \mathrm{mL} /$ day, respectively [9].

There was no statistically significant difference in the pharmacokinetics of pegaspargase with respect to sex [9]. Pegaspargase dosage adjustments are not required in patients with renal or hepatic impairment. However, pegaspargase is contraindicated in patients with severe hepatic impairment $[8,9]$. The pharmacokinetics of pegaspargase have not been investigated in patients aged $>65$ years [9].

\section{Therapeutic Efficacy of Pegaspargase}

The efficacy of IV or IM pegaspargase in patients with newly diagnosed ALL (Sect. 3.1) or relapsed ALL with hypersensitivity to $E$. coli L-asparaginase (Sect. 3.2) has been extensively examined in wide range of clinical trials, with the majority of the trials being primarily designed to evaluate the safety (including immunogenicity) or pharmacokinetics of pegaspargase $[9,12,15-21]$. Some data are available as abstracts $[16,20]$. Discussion in this section focuses on the trials relevant to the use of approved dosages of pegaspargase (Sect. 5) in the indicated patient population. Unless otherwise specified, efficacy analyses were conducted in the intent-to-treat population and pegaspargase was administered as a component of multiagent chemotherapy (generally containing cytarabine, vincristine, daunorubicin, methotrexate and prednisone/ dexamethasone) [12, 15-24].

\subsection{Newly Diagnosed Acute Lymphoblastic Leukaemia}

\subsubsection{Paediatric Population}

3.1.1.1 The CCG-1962 Trial The CCG-1962 trial compared the efficacy of pegaspargase to that of $E$. coli $\mathrm{L}$-asparaginase in paediatric patients (aged 1-9 years) with newly diagnosed standard risk (SR) ALL [11, 17]. Eligible patients had an initial white blood cell (WBC) count of $<50,000 / \mu \mathrm{L}$ and $\leq 25 \%$ L3 blasts. Patients with massive lymphadenopathy, massive splenomegaly, and/or a large mediastinal mass at diagnosis, or those with CNS or testicular leukaemia were also eligible [11, 17].

CCG-1962 consisted of a 4-week induction phase, followed by two 8 -week delayed intensification phases (DI 1 and DI 2) [11, 17]. Patients were randomized to receive IM pegaspargase $2500 \mathrm{IU} / \mathrm{m}^{2}(n=59)$ or $E$. coli $\mathrm{L}$-asparaginase $6000 \mathrm{IU} / \mathrm{m}^{2}(n=59)$ as a part of a multi-agent chemotherapeutic regimen. Pegaspargase was administered on day 3 of each treatment phase and $E$. coli L-asparaginase was administered three times weekly for nine doses during the induction phase and for six doses during each of the delayed intensification phases [11, 17].

Demographic and baseline characteristics were largely similar between the pegaspargase and the E. coli L-asparaginase groups, although some notable differences were evident, including the proportion of patients aged 1-2 years (34 vs. $19 \%$ ), platelet counts $<50,000 / \mu \mathrm{L}(51$ vs. $34 \%$ ) or those with equivocal CNS disease (15 vs. $7 \%$ ) [11, 17].

CCG-1962 was an immunogenicity study, where the incidence of anti-asparaginase antibodies was evaluated as a primary endpoint (see results in Sect. 4) [8, 9, 17]. In CCG-1962, the efficacy of pegaspargase was determined by demonstration of a comparable duration and magnitude of asparagine depletion in the pegaspargase and the E. coli $\mathrm{L}$-asparaginase groups. The protocol-specified goal was to achieve depletion of serum asparagine levels to $\leq 1 \mu \mathrm{mol} / \mathrm{L}$ $[8,9,17]$. Other endpoints included asparaginase activity, bone marrow responses and event-free survival (EFS) [11].

During all three phases of treatment at the protocol-specified time points, the proportion of patients achieving the goal of an asparagine depletion level of $\leq 1 \mu \mathrm{mol} / \mathrm{L}$ was similar between the treatment groups $[8,9,17]$. In each treatment phase, asparagine concentrations in plasma were moderately $(1-10 \mu \mathrm{mol} / \mathrm{L})$ or completely $(\leq 1 \mu \mathrm{mol} / \mathrm{L})$ depleted in majority of pegaspargase or $E$. coli L-asparaginase recipients within 4 days of the first dose and these decreased levels were sustained for $\approx 3$ weeks. Asparagine levels in CSF during the induction phase decreased in a similar manner to that 
seen with asparagine levels in plasma $[8,9,17]$. The median CSF asparagine levels were 1.17 and $1.06 \mu \mathrm{mol} / \mathrm{L}$ on day 7 in the pegaspargase and E. coli $\mathrm{L}$-asparaginase groups and were $<1 \mu \mathrm{mol} / \mathrm{L}$ by day 28 in both groups [17]. Depletion of CSF asparagine levels may be considered as a surrogate marker to evaluate the efficacy of pegaspargase for the treatment of CNS leukaemia [11].

Pegaspargase showed adequate asparaginase activity over a longer time period than $E$. coli $\mathrm{L}$-asparaginase, although serum asparaginase levels did not appear to correlate with asparagine depletion [17]. On day 21 of each of the DI 1 and 2 phases, the predefined target threshold of asparaginase activity $>0.1 \mathrm{IU} / \mathrm{mL}$ (i.e. activity level considered adequate for asparagine depletion) was seen in $91-95 \%$ of pegaspargase recipients (vs. 19-22\% with E. coli L-asparaginase) [11].

There was a statistically significant $(p \leq 0.05)$ faster clearance of lymphoblasts in bone marrow at day 7 and day 14 in the pegaspargase than in the E.coli L-asparaginase groups during the induction phase [11]. In addition, the EFS rates were similar between the treatment groups (Table 1); however, CCG-1962 was underpowered to detect the difference in EFS and the analysis was descriptive only [9, 11, 17]. After $\approx 3$ years' follow-up, seven of 59 patients in the pegaspargase group and eight of 59 patients in the E. coli L-asparaginase group relapsed [11].

3.1.1.2 The DFCl 05-001 Trial The efficacy of pegaspargase as a first-line treatment was further supported by the DFCI 05-001 trial in paediatric patients (aged 1-18 years) with newly diagnosed ALL [12]. DFCI 05-001 was a toxicity study and efficacy was evaluated as secondary outcomes. Predefined efficacy endpoints included disease-free survival (DFS), serum asparaginase activity and health-related quality of life (HR-QOL) [12].

Based on their baseline characteristics (e.g. WBC count, age, CNS status and immunophenotype), eligible patients were assigned to an initial risk group (i.e. SR or HR) and received a multi-agent induction chemotherapy for 32 days, with IV pegaspargase $2500 \mathrm{IU} / \mathrm{m}^{2}$ administered on day 7 $(n=551)$ [12]. Patients who achieved CR (i.e. $<5 \%$ marrow blasts and evidence of normal haemopoiesis, no extramedullary disease, and peripheral blood counts recovery) after the induction regimen were then randomized to receive 15 doses

Table 1 Efficacy of pegaspargase in patients with newly diagnosed acute lymphoblastic leukaemia

\begin{tabular}{|c|c|c|c|}
\hline Study & Age range (years) & Treatment $^{\mathrm{a}}\left(\right.$ no. of $\left.\mathrm{pts}^{\mathrm{b}}\right)$ & Clinical outcomes (\% of pts) \\
\hline \multicolumn{4}{|l|}{ Paediatric population } \\
\hline CCG-1962 [9] & $1-9$ & $\begin{array}{l}\text { PEG (59); } \\
\text { L-asp (59) }\end{array}$ & 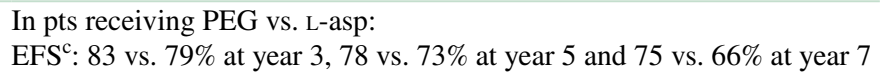 \\
\hline DFCI 05-001 [12] & $1-18$ & $\begin{array}{l}\text { PEG (232); } \\
\text { L-asp (231) }\end{array}$ & $\begin{array}{l}\text { In pts receiving PEG vs. L-asp: } \\
\text { 5-year DFS }{ }^{\text {d: }} 90 \text { vs. } 89 \% \text {; 5-year OS: } 96 \text { vs. } 94 \%\end{array}$ \\
\hline \multicolumn{4}{|l|}{ Mixed age population } \\
\hline AALL07P4 $[9,15]$ & $1-<31$ & PEG (50) & 4-year EFS $:$ : 82\%; 4-year OS: $90 \%$ \\
\hline DFCI 11-001 [9] & $1-<22$ & PEG (91) & 1-year EFS $: 98 \% ; 1$-year OS: $100 \%$ \\
\hline Douer et al. [18] & $18-57$ & PEG (51) & $\mathrm{CR}^{\mathrm{e}}:$ 96\%; 7-year DFS ${ }^{\mathrm{d}}: 58 \% ; 7$-year OS: $51 \%$, \\
\hline Rytting et al. [21] & $12-40$ & PEG (106) & $\mathrm{CR}^{\mathrm{e}}:$ 93\%; 5-year CRD: 53\%; 5-year OS: 60\% \\
\hline DeAngelo et al. [16] & $18-50$ & PEG (110) & $\mathrm{CR}^{\mathrm{e}}: 89 \% ; 3$-year DFS ${ }^{\mathrm{d}, \mathrm{f}}: 73 \% ; 3$-year OS: 75\% \\
\hline CALGB 10403 [23] & $17-39$ & PEG (295) & $\begin{array}{l}\text { Co-primary endpoints: } \mathrm{CR}^{\mathrm{e}}: 89 \% \text {; 3-year EFS }: 59 \% ; 3 \text {-year DFS }{ }^{\mathrm{d}, \mathrm{f}}: 66 \% \text {; } \\
\text { 3-year OS: } 73 \%\end{array}$ \\
\hline Gokbuget et al. [20] & $15-35$ & $\begin{array}{l}\text { PEG (887); } \\
\text { L-asp (642) }\end{array}$ & $\begin{array}{l}\text { In pts receiving PEG vs. L-asp: } \\
\text { CR }^{\text {e }}: 91 * \text { vs. } 88 \% \text {; } 5 \text {-year RD: } 61 * \text { vs. } 49 \% \text {; } 5 \text {-year OS: } 65 * \text { vs. } 46 \%\end{array}$ \\
\hline Ribera et al. [22] & $18-60$ & $\begin{array}{l}\text { PEG (35); } \\
\text { L-asp (91) }\end{array}$ & $\begin{array}{l}\text { In pts receiving PEG vs. L-asp: } \\
\text { CR }^{\text {e: }} 86 \text { vs. } 86 \% \text {; } 3 \text {-year DFS }{ }^{\text {d }}: 58 \text { vs. } 40 \% \text {; } 3 \text {-year OS: } 57 \text { vs. } 60 \%\end{array}$ \\
\hline
\end{tabular}

$C R$ complete remission after treatment, $C R D$ complete remission duration, $D F S$ disease-free survival, $E F S$ event-free survival, L-asp native $E$. coli asparaginase, $O S$ overall survival, $P E G$ pegaspargase, pts patients, $R D$ remission duration

$* p<0.001$ vs. L-asp

${ }^{a}$ Administered as a component of Children's Cancer Group [9, 15, 18, 23], Dana-Farber Cancer Institute [9, 12, 16], augmented Berlin-Frankfurt-Münster [21], German Multicentre Study Group for ALL [20] or Programa Español de Tratamientos en Hematología [22] protocols

${ }^{\mathrm{b}}$ Intent-to-treat population, some results [9, 16, 23] are based on evaluable pts

${ }^{\mathrm{c}}$ Defined as time from randomization to first event, including induction death, no induction response, relapse at any site or second malignant neoplasm [11, 23]; DFCI 11-001 [40], AALL07P4 [41] and CALGB 10403 [23] also regarded remission death as an EFS event

${ }^{\mathrm{d}}$ DFS events included relapse at any site, second malignant neoplasm or death during remission

${ }^{\mathrm{e}}$ Defined as $<5 \%$ marrow blasts in the bone marrow, no extramedullary disease and normal peripheral blood counts

${ }^{\mathrm{f}}$ Based on evaluable pts who achieved CR ( $n=90$ [16] and $n=263$ [23]) 
of IV pegaspargase $2500 \mathrm{IU} / \mathrm{m}^{2}$ every 2 weeks or 30 doses of IM $E$. coli $\mathrm{L}$-asparaginase $25,000 \mathrm{IU} / \mathrm{m}^{2}$ weekly during the 30 -week post-induction treatment period, starting at week 7 after study entry. Randomization was stratified by final risk group [i.e. SR, HR or very high risk (VHR)], based on minimal residual disease (MRD) and cytogenetics. Patients who achieved CR but declined to be randomized were directly assigned to the $E$. coli L-asparaginase group $(n=42)$, which is not discussed further. During the post-induction treatment period, patients who develop grade $\geq 2$ hypersensitivity reactions to $E$. coli $\mathrm{L}$-asparaginase were switched to IM pegaspargase $\left(2500 \mathrm{IU} / \mathrm{m}^{2}\right.$ weekly) and if they experienced a second hypersensitivity reaction, they were switched to twiceweekly IM Erwinia-derived asparaginase $25,000 \mathrm{IU} / \mathrm{m}^{2}$. Similarly, patients who developed grade $\geq 2$ hypersensitivity reactions to pegaspargase were switched to Erwinia-derived asparaginase [12].

The pegaspargase and E. coli $\mathrm{L}$-asparaginase groups were generally balanced in terms of baseline clinical and demographic characteristics [12]. At baseline, most patients were aged $<10$ years $(74 \%)$, and had a WBC count $<50,000 / \mu \mathrm{L}$ $(81 \%)$, a B cell immunophenotype (87\%) and no blast cells in CSF (76\%); 52, 37 and $11 \%$ of patients were classified as SR, HR and VHR as their final ALL risk group, respectively. Of the 463 randomized patients, 20 patients in the pegaspargase group and 21 patients in the $E$. coli $\mathrm{L}$-asparaginase group relapsed. A significantly $(p=0.015)$ higher proportion of pegaspargase than $E$. coli $\mathrm{L}$-asparaginase recipients completed all 30 weeks of treatment (82 vs. 74\%). [12].

After a median follow-up of 6 years, 5-year DFS and OS rates in patients receiving pegaspargase or E.coli L-asparaginase were not significantly different (Table 1) [12]. Furthermore, between the treatment groups, there was no statistically significant difference in DFS rates within patient subsets defined by baseline characteristics (e.g. age, sex, immunophenotype, WBC), final risk group classification or MRD status after induction [12].

At each post-induction time point (i.e. weeks 5, 11, 17, 23 and 29), both the median nadir serum asparaginase activity $(0.717-0.774$ vs. $0.084-0.105 \mathrm{IU} / \mathrm{mL})$ and the proportion of patients with predefined target nadir serum asparaginase activity of $\geq 0.1 \mathrm{IU} / \mathrm{mL}$ (94-100 vs. $43-51 \%$ ) were significantly $(p<0.0001)$ higher in pegaspargase than $E$. coli L-asparaginase recipients [12]. Moreover, the proportion of patients with at least one post-induction nadir serum asparaginase activity of $\geq 0.1 \mathrm{IU} / \mathrm{mL}$ was significantly ( $p<$ 0.0001 ) higher in pegaspargase than $E$. coli L-asparaginase recipients (99 vs. $71 \%$ ). The proportion of patients with undetectable nadir serum asparaginase activity (suggestive of silent inactivation) at each time point in the respective treatment groups was $0-3$ versus $6-12 \%$ [12].

DFCI 05-001 also evaluated the impact of pegaspargase $(n=105)$ or $E$. coli $\mathrm{L}$-asparaginase $(n=97)$ treatment on HR-QOL, as assessed by PedsQL surveys (consisted of six domains) [12]. Significantly ( $p \leq 0.03)$ greater improvements in treatment- or procedural-related anxiety from both parent-proxy and patient reports were observed in pegaspargase than E. coli $\mathrm{L}$-asparaginase recipients. Scores for other domains (e.g. emotional functioning, pain and hurt, general or rest fatigue, sleep) did not differ significantly between the treatment groups [12].

\subsubsection{Mixed Age Population}

Pegaspargase was used as the active comparator (to calaspargase pegol) in two randomized, open-label studies (AALL07P4 pilot study [15] and the phase II DFCI 11-001 trial [9]); DFCI 11-001 is currently ongoing [9]. Data for the pegaspargase arm are discussed here. These studies enrolled paediatric, adolescent and young adult patients with HR B-precursor ALL (AALL07P4 [15]) or those with newly diagnosed B- or T-cell ALL, regardless of risk group (DFCI 11-001 [9]).

A maximum of 12 doses of IV pegaspargase $2500 \mathrm{IU} / \mathrm{m}^{2}$ was administered during the induction, consolidation, delayed intensification, and interim maintenance phases in AALL07P04 [15]; IV pegaspargase $2500 \mathrm{IU} / \mathrm{m}^{2}$ was administered on day 7 during the induction phase in DFCI 11-001, and then biweekly for 30 weeks' post-induction treatment period [9]. In both trials, $\geq 80 \%$ of patients treated with pegaspargase had negative/low MRD on day 29 (AALL07P4) and on day 32 (DFCI 11-001) of the induction phase. EFS and OS rates with pegaspargase treatment in both trials were $\geq 82 \%$ (Table 1) [9].

The feasibility of treating adolescents and adults (aged $\geq 12$ years) with newly diagnosed ALL with paediatric-inspired ALL protocols containing pegaspargase was also demonstrated in four $(n=50-295)$ open-label trials [16, $18,21,23]$. Enrolled patients had a newly diagnosed SR or HR ALL and received a multi-agent paediatric-inspired ALL protocol that included multiple doses of pegaspargase 2000 or $2500 \mathrm{IU} / \mathrm{m}^{2}$. The dosing interval between each pegaspargase administration was adjusted appropriately to reduce the incidence of pegaspargase-related toxicities. Efficacy analyses included the end-induction CR rate, EFS, DFS or complete remission duration (CRD), and/or OS rates. Pegaspargase-containing regimens were associated with a CR rate of $89-96 \%$ after $\approx 4$ weeks' treatment and a 5-year CRD rate of 53\% (Table 1). These findings are supported by EFS, DFS and OS outcomes (Table 1) [16, 18, 21, 23].

Improved clinical outcomes with pegaspargase were further supported by results from a large cohort of adolescents and adults with SR, HR or VHR ALL from the GMALL 07/03 trial [20, 25]. Enrolled patients received the pegaspargase-containing optimized paediatric-inspired German Multicentre Study Group for ALL (GMALL) 
protocol and the efficacy outcomes (CR, remission duration and OS) were compared with a cohort of patients with similar baseline characteristics from the GMALL 05/93 trial in which patients received the $E$. coli $\mathrm{L}$-asparaginasecontaining standard GMALL protocol. Relative to the standard GMALL protocol, patients receiving the pegaspargasecontaining optimized GMALL protocol had significantly $(p<0.001)$ improved CR, remission duration and OS rates (Table 1). However, improved clinical outcomes cannot be solely attributed to the use of pegaspargase, but may be a result of an optimized GMALL protocol in general [20, 25].

The association between the efficacy of pegaspargase and asparagine depletion was demonstrated in the CALGB 9511 study in patients aged 17-71 years with newly diagnosed ALL ( $n=85$ evaluable) [24]. Based on a univariate analysis, those who achieved asparagine depletion (defined as asparaginase activity $>0.03 \mathrm{IU} / \mathrm{mL}$ for consecutive 14 days) following 1-4 doses of pegaspargase $2000 \mathrm{U} / \mathrm{m}^{2}$ (each dose was capped at $3750 \mathrm{U}$ ) during the induction and the first intensification phases had a significantly $(p \leq 0.012)$ superior DFS [hazard ratio (HR) 2.21 ; 95\% CI 1.19-4.13] and OS (HR 2.37; 95\% CI 1.38-4.09) than those who failed to achieve asparagine depletion [24].

A retrospective analysis in Chinese patients (aged $\geq 14$ years) with newly diagnosed ALL $(n=122)$ evaluated the efficacy of pegaspargase and $E$. coli $\mathrm{L}$-asparaginase when each drug was administered as a part of induction ALL regimen [19]. In this analysis, there were no significant between-group differences in terms of CR rate (96 vs. 91\%), median relapse-free survival (RFS; 10 vs. 9 months) and OS (14 vs. 16 months). However, in patients aged $<35$ years, RFS was significantly longer in the pegaspargase than in the E. coli $\mathrm{L}$-asparaginase group (11 vs. 9 months; $p=0.037$ ). Moreover, a significantly lower incidence of CNS leukaemia during the consolidation phase was reported in pegaspargase than E. coli L-asparaginase recipients (11 vs. $28 \% ; p=0.028$ ) [19]. Similarly, in an observational study conducted in Spanish patients with newly diagnosed HR Philadelphia chromosome-negative ALL, there were no statistically significant differences between the pegaspargase $(n=35)$ and $E$. coli L-asparaginase $(n=91)$ groups in terms of CR, DFS and OS rates, when the treatment was given as a part of ALL regimen during the induction and consolidation phases (Table 1) [22]. This study, however, was not sufficiently powered to detect the between-group differences [22].

\subsection{Relapsed Acute Lymphoblastic Leukaemia with Hypersensitivity to $E$. coli L-asparaginase}

This section focuses on data obtained from patients with relapsed ALL who were hypersensitive to $E$. coli L-asparaginase, as evaluated in the key phase II ASP-304 trial [26].
ASP-304 included patients (aged $<21$ years) with ALL who were in second bone marrow relapse (i.e. $\mathrm{M}_{3} ;>25 \%$ marrow blasts) and those had been treated with $E$. coli L-asparaginase. Patients with a life expectancy $<4$ weeks, or those with inadequate hepatic or renal function were excluded [26].

Of 76 enrolled patients, 42 patients had a prior hypersensitivity to E. coli L-asparaginase (11 patients also had hypersensitivity to Erwinia-derived asparaginase) and were directly assigned to the pegaspargase group; two patients without hypersensitivity to $E$. coli L-asparaginase were assigned to the pegaspargase group as they were labeled incorrectly [26]. Assigned patients received IM pegaspargase $2500 \mathrm{IU} / \mathrm{m}^{2}$ on days 1 and 15 during the reinduction phase, based on the findings from phase I data in hypersensitive patients. Pegaspargase was administered in combination with a standard ALL reinduction regimen that consisted of vincristine and prednisone. After completion of the 36-day study period, responders could continue receiving IM pegaspargase $2500 \mathrm{IU} / \mathrm{m}^{2}$ biweekly as a monotherapy or as a combination therapy with other standard anti-leukaemic agents [26].

At entry, the mean age of hypersensitive patients was 8.55 years [26]. Clinical response to pegaspargase treatment was evaluated by bone marrow aspirate, peripheral blood, and CSF examinations on day 29. Response criteria for bone marrow remission included CR (M1; $\leq 5 \%$ marrow blasts), partial remission (PR; $\mathrm{M} 2 ;>5$ to $\leq 25 \%$ marrow blasts), no response (M3; $>25 \%$ marrow blasts) and progressive disease (PD; $>25 \%$ increase in marrow or peripheral blood blasts, or rapidly progressive organomegaly). Of 42 hypersensitive patients, $38 \%$ achieved CR, $12 \%$ achieved PR and $48 \%$ of patients did not respond to the treatment or had PD; one patient was not evaluable [26].

When data from ASP-304 were pooled with four other small $(n<20)$ open-label trials (ASP-001, ASP-201A, ASP302 and ASP-400), the results were generally consistent with that observed in ASP-304 [9, 26]. These trials included patients aged 1-73 years with relapsed hematological malignancies [13]. In total, 65 patients with relapsed ALL and prior hypersensitivity to $E$. coli L-asparaginase (most of whom were aged $<21$ years) received IM or IV pegaspargase 2000 or $2500 \mathrm{IU} / \mathrm{m}^{2}$ biweekly as monotherapy or in combination with other anti-leukaemic agents; one patient received IV pegaspargase 250 and $500 \mathrm{IU} / \mathrm{m}^{2}$ biweekly. Of these 65 patients, $46 \%$ achieved CR, $11 \%$ achieved PR and $2 \%$ showed haematological improvement [9].

\section{Tolerability of Pegaspargase}

Extensive evidence from clinical trials and post-marketing use $(n>100,000)$ indicate that pegaspargase has a well established safety profile [13]. IV or IM pegaspargase had 
a manageable tolerability profile in children or adults with ALL, based on data from clinical trials, including DFCI 11-001, AALL07P04 and CCG-1962 (Sect. 3) [8, 9]. The most common (incidence $\geq 10 \%$ ) adverse events (AEs) with pegaspargase treatment included hepatotoxicity (increased alanine aminotransferases, aspartate aminotransferases and/ or bilirubin), increased activated partial thromboplastin time, hypertriglyceridaemia, hyperglycaemia and febrile neutropenia [9]. The most common ( $>5 \%)$ grade $\geq 3$ AEs included hepatotoxicity (elevated transaminases, increased bilirubin and hypoalbuminemia), febrile neutropenia, hypertriglyceridemia, hyperglycaemia, pancreatitis, abnormal coagulopathy, embolic and thrombotic events, and hypersensitivity [8].

The tolerability profile of pegaspargase was generally consistent with that seen with $E$. coli $\mathrm{L}$-asparaginase in trials in paediatric patients (CCG-1962 [17] and DFCI 05-001 [12]) and in adult patients [19] with ALL. For instance, in DFCI 05-001 (Sect. 3.1.1.2), the incidence of asparaginaserelated AEs (hypersensitivity reactions, pancreatitis and thrombotic or bleeding complications) [primary endpoint] in the pegaspargase group was not significantly different to that reported in the E. coli $\mathrm{L}$-asparaginase group (28 vs. $26 \%$ ) [12]. In the respective groups, the incidence of grade $\geq 2$ pancreatitis (12 vs. $10 \%$ ), grade $\geq 2$ thrombosis or bleeding (7 vs. 10\%) and hypersensitivity reaction of any grade (12 vs. 9\%) did not show any statistically significant differences. The most common grade $\geq 3$ AEs in pegaspargase recipients were bacterial or fungal infections ( 20 vs. $22 \%$ with E. coli L-asparaginase) and hypersensitivity reactions (6 vs. $3 \%$ ). One treatment-related death was reported in each treatment group (each due to CNS haemorrhage and infection) [12]. Similarly, in the retrospective analysis in adult patients with ALL (Sect. 3.1.2), the incidence hypersensitivity, grade 3 or 4 hepatotoxicity, renal function damage, pancreatic lesions and bleeding events in the pegaspargase group during the induction phase was not significantly different to that reported in the E. coli L-asparaginase group [19].

Despite the potential increased risk of asparaginaserelated toxicities in adult patients with ALL (Sect. 3.1.2) [18], a reaL-world safety analysis set in the US in newly diagnosed ALL showed a generally similar safety profile with pegaspargase in adolescent/adult patients $(n=76)$ compared with paediatric patients $(n=1247)$, with the exception of hepatotoxicity and pancreatic dysfunction (Fig. 1) [27]. In a separate retrospective review, it was considered that raised body mass index may increase the risk of hepatotoxicity (abstract [28]).

The nature of pegaspargase-related AEs occurring in patients with relapsed ALL and hypersensitivity to $E$. coli $\mathrm{L}$-asparaginase were generally consistent with those observed in patients newly diagnosed ALL, with the exception of hypersensitivity reactions (Sect. 4.1) [8, 9]. In ASP304 in patients with relapsed ALL (Sect. 3.2), all patients tolerated two doses of pegaspargase during the reinduction phase and 27 patients continued to receive pegaspargase during the maintenance treatment phase for up to 35 months without clinical reactions [26]. Of 40 hypersensitive patients receiving pegaspargase, $83 \%$ reported a total of 75 AEs of any grade that were considered treatment-related, with the most common $(>5 \%)$ being increased serum alanine aminotransferase (45\%), hypoproteinemia (40\%), decreased fibrinogen $(30 \%)$, increased partial thromboplastin time (15\%), hyperbilirubinemia (15\%), fever (8\%) and hypersensitivity reactions (numerical data not reported) [13].

Appropriate monitoring, treatment interruption, modification or discontinuation of pegaspargase may be necessary in patients experiencing asparaginase-related toxicities (including hepatotoxicity, pancreatitis and thrombosis); supportive or appropriate treatment may also be required $[8,9]$. For instance, patients should be monitored for liver function, serum glucose/amylase/lipase levels and coagulation parameters throughout the treatment period. Moreover, pegaspargase should be discontinued in the event of pancreatitis (can be fatal if left untreated), serious hepatotoxicity or serious thrombosis $[8,9]$. Patients with serious hepatotoxicity or pancreatitis can be provided with supportive care $[8,13]$ and those with thrombosis can be treated with low-molecularweight-heparin anticoagulation therapy [27].

As with all therapeutic proteins, there is a potential for immunogenicity with pegaspargase [8]. The primary endpoint in CCG-1962 (Sect. 3.1.1.1) was the incidence of protocol-specified high-titre anti-asparaginase antibodies (i.e. a serum antibody to negative control ratio of $>2.5$ ) at the DI 1 phase using ELISA [11]. It should be noted that CCG-1962 was underpowered to detect the between-group difference, as it was incorrect to assume that $50 \%$ of patients receiving native E.coli $\mathrm{L}$-asparaginase would develop high-titre

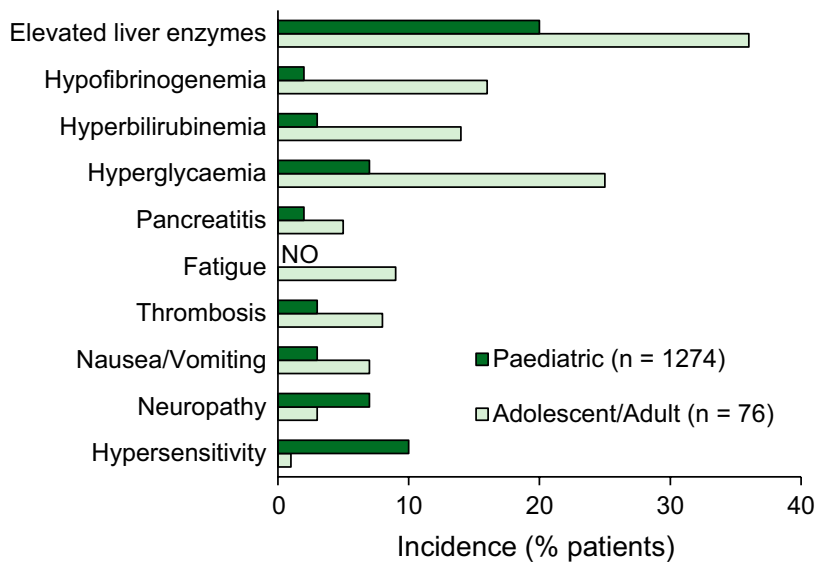

Fig. 1 The incidence of most common ( $>5 \%$ in any of the age group) grade 3 or 4 pegaspargase-related toxicities occurring in adolescent/ adult (aged 14-68 years) and paediatric (aged 0-18 years) patients with acute lymphoblastic leukaemia [27]. NO not obtained 
antibodies. The incidence of high-titre anti-asparaginase antibodies in patients receiving pegaspargase $(n=49)$ or E. coli $\mathrm{L}$-asparaginase $(n=46)$ during the DI 1 phase was 6 versus $15 \%$ (data on file). Overall, $12 \%$ of pegaspargase versus $28 \%$ of E.coli $\mathrm{L}$-asparaginase recipients in the respective groups had high-titre anti-asparaginase antibodies at any time during the study period [17]. In addition, in ASP-304 in relapsed ALL patients (Sect. 3.2), high-titre anti-asparaginase antibodies (i.e. 3 in an ordinal scale of $0-3$ vs. reference standard) with pegaspargase treatment was more prevalent in hypersensitive patients $(n=30)$ than nonhypersensitive patients $(n=12)$ [87 vs. $42 \%$ ] [26].

\subsection{Hypersensitivity Reactions}

Patients receiving pegaspargase may experience hypersensitivity reactions (including anaphylaxis, bronchospasm, hypotension, laryngeal oedema, local erythema or swelling, rash and urticaria) and the risk of serious hypersensitivity reaction is particularly high in patients with known hypersensitivity to $E$. coli L-asparaginase [8, 9]. In studies discussed in Sect. 3.1, where specified, 1-23\% of pegaspargase recipients experienced hypersensitivity reactions of any grade and grade $\geq 3$ hypersensitivity reactions were seen in $2-10 \%$ of patients $[8,12,15-19,21,23]$. In patients with relapsed ALL and prior hypersensitivity to $E$. coli L-asparaginase $(n=62)$, hypersensitivity reactions were reported in $32 \%$ of patients (vs. $10 \%$ of 112 nonhypersensitive patients), with $8 \%$ being grade $\geq 3$ [8].

The incidence of hypersensitivity reactions with pegaspargase across all treatment phases was generally similar to [17] or did not differ significantly from $[12,19]$ that observed with $E$. coli $\mathrm{L}$-asparaginase in patients with newly diagnosed ALL. However, hypersensitivity reactions with pegaspargase mostly occurred at an earlier exposure compared with E. coli L-asparaginase [12]. In DFCI 05-001, of the reported 49 hypersensitivity reactions, 25 of 28 reactions in the pegaspargase group occurred after the first or second post-induction dose compared with 2 of 21 reactions in the E. coli $\mathrm{L}$-asparaginase group. Hypersensitivity was diagnosed after a median of one and five post-induction doses in the respective groups [12].

Other factors that may increase the likelihood of patients experiencing hypersensitivity reactions with pegaspargase treatment include the patient's ALL risk group and the route of administration [29-32]. For instance, SR ALL was associated with a significantly $(p \leq 0.025)$ lower risk of hypersensitivity reactions than HR ALL in a population-based study (OR 7.8; 95\% CI 1.34-45.1) [30], and a chart review (OR 3.36; 95\% CI 1.16-9.72) [31], with similar results also reported in a meta-analysis (2.5 vs. $19.1 \%$; $95 \%$ CIs did not overlap) [29]. A recent analysis of toxicity data from six COG trials $(n=16,534)$ showed that the incidence of grade $\geq 3$ hypersensitivity reactions was significantly $(p<0.0001)$ higher with IM than IV pegaspargase when all pegaspargase doses during the treatment phases (5.4 vs. $3.2 \%)$ and when second and third doses of pegaspargase (10.1 vs. $5.0 \%)$ were evaluated [32].

\section{Dosage and Administration of Pegaspargase}

In the USA, pegaspargase as a component of a multi-agent chemotherapy is indicated for the first-line treatment of paediatric and adult patients with ALL, as well as for the treatment of paediatric and adult patients with ALL and hypersensitivity to $E$. coli L-asparaginase [8]. In the EU, pegaspargase is indicated as a component of antineoplastic combination therapy for ALL in paediatric (aged $\leq 18$ years) and adult patients [9]. Pegaspargase is available as a clear and colourless solution and is provided in a single-use vial containing $3750 \mathrm{IU}$ in a $5 \mathrm{~mL}$ solution for IV infusion or IM injection [8,9]. Since 2018, pegaspargase has also been available as a lyophilized powder for reconstitution in the EU [9]. The recommended dosage of pegaspargase is different in the US and EU and in patients aged $\leq 21$ years and those aged $>21$ years (please see supplementary information for specific details) $[8,9]$. To monitor the efficacy of pegaspargase treatment, trough asparaginase activity level may be measured and a switch to a different asparaginase preparation could be considered if the activity fails to reach target levels [9]. Dose interruption/modification or discontinuation of pegaspargase may be required for the management of AEs $[8,9]$. Local prescribing information should be consulted for full details regarding the use of pegaspargase, including administration instructions, contraindications, potential drug interactions, warnings, precautions and management of tolerability issues.

\section{Place of Pegaspargase in the Management of Acute Lymphoblastic Leukaemia}

The efficacy of pegaspargase for the treatment of ALL is well established and it has been used to treat more than 100,000 patients worldwide, including ALL patients with hypersensitivity reaction to $E$. coli asparaginase [13]. Currently, pegaspargase is recommended as a core component of ALL regimens in the NCCN and the ESMO guidelines $[1,2]$. NICE guidance also recommends pegaspargase as an option for the treatment of untreated, newly diagnosed ALL in children and adults [33]. With E. coli L-asparaginase being no longer available in the USA [1], pegaspargase has replaced E. coli L-asparaginase in most of the ALL protocols $[1,4]$. 
Pegylation of L-asparaginase improves both the pharmacokinetic profile and immunogenicity of the enzyme without affecting the enzymatic properties of L-asparaginase [13]. Pegaspargase has a longer elimination half-life than E. coli $\mathrm{L}$-asparaginase (Sect. 2.2), thereby offering less frequent administration by replacing six or nine doses of $E$. coli $\mathrm{L}$-asparaginase with one single dose of pegaspargase [17]. In the CCG-1962 trial, a numerically lower proportion of pegaspargase than $E$. coli L-asparaginase recipients had high-titre anti-asparaginase antibodies at the DI 1 phase (Sect. 3). However, these comparisons should be interpreted with caution as the detection of antibody formation is highly dependent on the sensitivity and specificity of the assay method and the positive results of the assay may be influenced by several other factors (e.g. sample handling, concomitant medications) [8]. Nevertheless, pegaspargase was associated with reduced immunogenicity, according to data obtained from several studies [13]. Anti-asparaginase antibodies may induce hypersensitivity reactions and/or loss of asparaginase activity (Sect. 1), although there is insufficient information to determine this currently [8].

The efficacy of pegaspargase was evaluated in a wide range of clinical trials (Sect. 3). The use of IM or IV pegaspargase as a component of multi-agent chemotherapy provided similar duration and magnitude of asparagine depletion, DFS and/or OS rates to that seen with E. coli L-asparaginase treatment in patients with newly diagnosed ALL (Sect. 3.1). Pegaspargase was also associated with greater improvements in treatment- or procedural-related anxiety than E.coli L-asparaginase (Sect. 3.1.1.2). Moreover, the feasibility of a pegaspargase-containing regimen was also demonstrated in several studies in adults with ALL, a population known to be more susceptible to asparaginaserelated toxicity (Sect. 3.1.2). Pegaspargase as a component of multi-agent chemotherapy was also effective for the treatment of paediatric and adult patients with relapsed ALL who were hypersensitive to $E$. coli $\mathrm{L}$-asparaginase (Sect. 3.2).

It is generally considered clinically relevant asparaginase activity should be maintained for optimal asparagine depletion [11, 12, 15, 24] and trough asparaginase activity level with pegaspargase treatment may be measured to monitor efficacy (Sect. 5) [9]. A higher proportion of ALL patients treated with IM or IV pegaspargase than E. coli L-asparaginase in clinical trials achieved asparaginase activity of $\geq 0.1 \mathrm{IU} / \mathrm{mL}$, a target concentration considered adequate for asparagine depletion; however, this did not translate to higher DFS or OS rates or greater asparagine depletion (Sect. 3.1.1). Of interest, the suggested target asparaginase activity to achieve sufficient asparagine depletion with pegaspargase varies across published studies (e.g. $\geq 0.03 \mathrm{IU} / \mathrm{mL}$ [24], $\geq 0.1 \mathrm{IU} / \mathrm{mL}[11,12]$ or $>0.4 \mathrm{IU} / \mathrm{mL}[15])$. The optimum target asparaginase activity to achieve maximum therapeutic benefit of pegaspargase remains to be fully elucidated.
Pegaspargase had a manageable tolerability profile in paediatric and adult patients with newly diagnosed ALL or those with relapsed ALL and hypersensitivity to E. coli L-asparaginase (Sect. 4). Of note, although the safety profile of pegaspargase in adults was generally consistent with that of $E$. coli $\mathrm{L}$-asparaginase and revealed no new safety concerns, a higher incidence of hepatotoxicity and pancreatic dysfunction with pegaspargase was reported in adult than paediatric patients with ALL.

Concerns have been raised about hypersensitivity issues with asparaginase treatment. The incidence of hypersensitivity reactions with pegaspargase across all treatment phases was generally consistent to that observed with $E$. coli $\mathrm{L}$-asparaginase in patients with newly diagnosed ALL (Sect. 4.1). However, the reactions appeared to occur at an earlier exposure during the post-induction phases with pegaspargase compared with E. coli L-asparaginase. Currently, there is no definite consensus on the management of patients who develop hypersensitivity reactions to $E$ coli asparaginase; physicians have a choice between switching to pegaspargase or Erwinia-derived asparaginase for ongoing treatment, depending on the protocol specification and preparation availability [34]. It was suggested that the use of pegaspargase during the induction phase may reduce the subsequent risk of hypersensitivity reaction, regardless of the asparaginase preparation used at the subsequent treatment phases $[6,12]$. In addition, the incidence of pegaspargase-associated hypersensitivity reaction was also more prevalent if pegaspargase was administered for the treatment of HR ALL, as an IM injection, or in patients with prior hypersensitivity to E. coli L-asparaginase (Sect. 4.1).

Pegaspargase was estimated to be a cost-effective option compared with $E$. coli $\mathrm{L}$-asparaginase for the first-line treatment of ALL in paediatric and adults patients [33, 35]. A cost-utility analysis in the UK over the lifetime horizon has shown that the strategy starting with pegaspargase followed by Erwinia-derived asparaginase in cases of hypersensitivity reaction was estimated to be more costeffective than the strategy starting with $E$. coli L-asparaginase or Erwinia-derived asparaginase in patients with newly diagnosed ALL, with a total cost savings of $£ 4741$ and a quality-adjusted life-years gain of 0.05 [35]. NICE guidance recommends pegaspargase as a cost-effective use of NHS resources for treating ALL in children, young people and adults with untreated, newly diagnosed disease [33]. A number of cost-minimization analyses in Europe are supportive of pegaspargase [36-39].

In conclusion, IM or IV administration of pegaspargase as a component of multi-agent chemotherapy has been proven to be an effective treatment, with a manageable tolerability profile, in paediatric and adult patients with ALL. Given the potentially reduced immunogenicity and more convenient dosage regimen over E. coli L-asparaginase, 
pegaspargase remains an important and effective treatment option for paediatric and adult patients with ALL, including those with hypersensitivity to $E$. coli L-asparaginase.

\section{Data Selection Pegaspargase: 514 records} identified

Duplicates removed

Excluded during initial screening (e.g. press releases; news reports; not relevant drug/indication; preclinical study; reviews; case reports; not randomized trial)

Excluded during writing (e.g. reviews; duplicate data; small patient number; nonrandomized/phase I/II trials)

Cited efficacy/tolerability articles

Cited articles not efficacy/tolerability

Search Strategy: EMBASE, MEDLINE and PubMed from 1946 to present. Clinical trial registries/databases and websites were also searched for relevant data. Key words were PEG-L-asparaginase, pegylated asparaginase, pegasparaginase, pegaspargase, Oncospar, acute lymphoblastic leukaemia. Records were limited to those in English language. Searches last updated 9 Apr 2019

Acknowledgements During the peer review process, the manufacturer of pegaspargase was also offered an opportunity to review this article. Changes resulting from comments received were made on the basis of scientific and editorial merit.

\section{Compliance with Ethical Standards}

Funding The preparation of this review was not supported by any external funding.

Conflicts of interest Young-A Heo, Yahiya Syed and Susan Keam are salaried employees of Adis/Springer, are responsible for the article content and declare no relevant conflicts of interest.

Open Access This article is distributed under the terms of the Creative Commons Attribution-NonCommercial 4.0 International License (http://creativecommons.org/licenses/by-nc/4.0/), which permits any noncommercial use, duplication, adaptation, distribution and reproduction in any medium or format, as long as you give appropriate credit to the original author(s) and the source, provide a link to the Creative Commons license and indicate if changes were made.

\section{References}

1. National Comprehensive Cancer Network. Acute lymphoblastic leukemia (NCCN clinical practice guidelines in oncology). 2018. http://www.nccn.org/. Accessed 4 Mar 2019.

2. Hoelzer D, Bassan R, Dombret H, et al. Acute lymphoblastic leukaemia in adult patients: ESMO clinical practice guidelines for diagnosis, treatment and follow-up. Ann Oncol. 2016;27(Suppl 5):v69-82.
3. Asselin BL. The three asparaginases: comparative pharmacology and optimal use in childhood leukemia. In: Kaspers GJL, Pieters R, Veerman AJP, editors. Drug resistance in leukemia and lymphoma III Advances in experimental medicine and biology. Boston: Springer; 1999. p. 621-9.

4. Pui CH, Evans WE. Treatment of acute lymphoblastic leukemia. N Engl J Med. 2006;354(2):166-78.

5. Silverman LB, Supko JG, Stevenson KE, et al. Intravenous PEGasparaginase during remission induction in children and adolescents with newly diagnosed acute lymphoblastic leukemia. Blood. 2010;115(7):1351-3.

6. Tong WH, Pieters R, Kaspers GJ, et al. A prospective study on drug monitoring of PEGasparaginase and Erwinia asparaginase and asparaginase antibodies in pediatric acute lymphoblastic leukemia. Blood. 2014;123(13):2026-33.

7. Pisal DS, Kosloski MP, Balu-Iyer SV. Delivery of therapeutic proteins. J Pharm Sci. 2010;99(6):2557-75.

8. Shire Pharmaceuticals. Oncaspar (pegaspargase): US prescribing information. 2019. http://www.fda.gov. Accessed 9 Apr 2019.

9. European Medicines Agency. Oncaspar (pegaspargase): summary of product characteristics. 2018. http://www.ema.europa.eu/. Accessed 9 Apr 2019.

10. Servier Pharmaceuticals. ASPARLASTM (calaspargase pegolmknl): US prescribing information. 2018. http://www.fda.gov. Accessed 9 Apr 2019.

11. Avramis VI, Sencer S, Periclou AP, et al. A randomized comparison of native Escherichia coli asparaginase and polyethylene glycol conjugated asparaginase for treatment of children with newly diagnosed standard-risk acute lymphoblastic leukemia: a children's cancer group study. Blood. 2002;99(6):1986-94.

12. Place AE, Stevenson KE, Vrooman LM, et al. Intravenous pegylated asparaginase versus intramuscular native Escherichia coli L-asparaginase in newly diagnosed childhood acute lymphoblastic leukaemia (DFCI 05-001): a randomised, open-label phase 3 trial. Lancet Oncol. 2015;16(16):1677-90.

13. European Medicines Agency. EPAR assessment report: oncaspar 2016. http://www.ema.europa.eu. Accessed 9 Apr 2019.

14. Wurthwein G, Lanvers-Kaminsky C, Hempel G, et al. Population pharmacokinetics to model the time-varying clearance of the pegylated asparaginase Oncaspar ${ }^{(\mathrm{R})}$ in children with acute lymphoblastic leukemia. Eur J Drug Metab Pharmacokinet. 2017;42(6):955-63.

15. Angiolillo AL, Schore RJ, Devidas M, et al. Pharmacokinetic and pharmacodynamic properties of calaspargase pegol Escherichia coli L-asparaginase in the treatment of patients with acute lymphoblastic leukemia: results from children's oncology group study AALL07P4. J Clin Oncol. 2014;32(34):3874-82.

16. DeAngelo DJ, Stevenson K, Neuberg DS, et al. A multicenter phase II study using a dose intensified pegylated-asparaginase pediatric regimen in adults with untreated acute lymphoblastic leukemia: a DFCI ALL consortium trial [abstract]. Blood. 2015;126(23):80.

17. Dinndorf PA, Gootenberg J, Cohen $\mathrm{MH}$, et al. FDA drug approval summary: pegaspargase $\left(\right.$ Oncaspar $\left.^{\circledR}\right)$ for the first-line treatment of children with acute lymphoblastic leukemia (ALL). Oncologist. 2007;12(8):991-8.

18. Douer D, Aldoss I, Lunning MA, et al. Pharmacokinetics-based integration of multiple doses of intravenous pegaspargase in a pediatric regimen for adults with newly diagnosed acute lymphoblastic leukemia. J Clin Oncol. 2014;32(9):905-11.

19. Liang J, Shi P, Guo X, et al. A retrospective comparison of Escherichia coli and polyethylene glycol-conjugated asparaginase for the treatment of adolescents and adults with newly diagnosed acute lymphoblastic leukemia. Oncol Lett. 2018;15(1):75-82.

20. Gökbuget N, Beck J, Brandt K, et al. Significant improvement of outcome in adolescents and young adults (AYAs) aged 
15-35 years with acute lymphoblastic leukemia (ALL) with a pediatric derived adult ALL protocol; results of 1529 AYAs in 2 consecutive trials of the german multicenter study group for adult ALL (GMALL) [abstract]. Blood. 2013;122(21):839.

21. Rytting ME, Jabbour EJ, Jorgensen JL, et al. Final results of a single institution experience with a pediatric-based regimen, the augmented Berlin-Frankfurt-Munster, in adolescents and young adults with acute lymphoblastic leukemia, and comparison to the hyper-CVAD regimen. Am J Hematol. 2016;91(8):819-23.

22. Ribera JM, Morgades M, Montesinos P, et al. Efficacy and safety of native versus pegylated Escherichia coli asparaginase for treatment of adults with high-risk, philadelphia chromosomenegative acute lymphoblastic leukemia. Leuk Lymphoma. 2018;59(7):1634-43.

23. Stock W, Luger SM, Advani AS, et al. A pediatric regimen for older adolescents and young adults with acute lymphoblastic leukemia: results of CALGB 10403. Blood. 2019;133(14):1548-59.

24. Wetzler M, Sanford BL, Kurtzberg J, et al. Effective asparagine depletion with pegylated asparaginase results in improved outcomes in adult acute lymphoblastic leukemia: cancer and leukemia group B Study 9511. Blood. 2007;109(10):4164-7.

25. Therapeutic Goods Administration. Extract from the clinical evaluation report for pegaspargase. 2017. http://www.tga.gov. au/. Accessed 9 Apr 2019.

26. Kurtzberg J, Asselin B, Bernstein M, et al. Polyethylene glycolconjugated L-asparaginase versus native L-asparaginase in combination with standard agents for children with acute lymphoblastic leukemia in second bone marrow relapse: a children's oncology group study (POG 8866). J Pediatr Hematol Oncol. 2011;33(8):610-6.

27. Stock W, Douer D, DeAngelo DJ, et al. Prevention and management of asparaginase/pegasparaginase-associated toxicities in adults and older adolescents: recommendations of an expert panel. Leuk Lymphoma. 2011;52(12):2237-53.

28. De Abrew KG, Richardson DS, Cook MA, et al. Hepatotoxicity with pegylated-asparaginase in acute lymphoblastic leukaemia is influenced by raised body mass index [abstract no. 249]. Br J Haematol. 2016;173 (Suppl 1):103.

29. Hasan H, Shaikh OM, Rassekh SR, et al. Comparison of hypersensitivity rates to intravenous and intramuscular PEG-asparaginase in children with acute lymphoblastic leukemia: a meta-analysis and systematic review. Pediatr Blood Cancer. 2017;64(1):81-8.

30. MacDonald T, Kulkarni K, Bernstein M, et al. Allergic reactions with intravenous compared with intramuscular pegaspargase in children with high-risk acute lymphoblastic leukemia: a population-based study from the maritimes, Canada. J Pediatr Hematol Oncol. 2016;38(5):341-4.

31. Abbott LS, Zakova M, Shaikh F, et al. Allergic reactions associated with intravenous versus intramuscular pegaspargase: a retrospective chart review. Paediatr Drugs. 2015;17(4):315-21.

32. Burke MJ, Devidas M, Maloney K, et al. Severe pegaspargase hypersensitivity reaction rates (grade $\geq 3$ ) with intravenous infusion vs. intramuscular injection: analysis of 54,280 doses administered to 16,534 patients on children's oncology group (COG) clinical trials. Leuk Lymphoma. 2018;59(7):1624-33.

33. National Institue for Health and Care Excellence. Technology appraisal guidance: pegaspargase for treating acute lymphoblastic leukaemia. 2016. http://www.nice.org.uk. Accessed 9 Apr 2019.

34. van der Sluis IM, Vrooman LM, Pieters R, et al. Consensus expert recommendations for identification and management of asparaginase hypersensitivity and silent inactivation. Haematologica. 2016;101(3):279-85.

35. Basu S, Lin PL, Saha V. The cost-effectiveness of pegaspargase for first-line treatment of acute lymphoblastic leukaemia: a costutility analysis. [abstract no. PCN180]. Value Health. 2017;20 (9):A444.

36. Delattre C, Lecureuil C, Staginnus U, et al. Cost-minimization analysis of pegaspargase and native $\mathrm{E}$. Coli asparaginase to treat children and adults with acute lymphoblastic leukemia in Belgium. [abstract no. PSY86]. Value Health. 2016;19(7):A590.

37. Faiella M, Lecureuil C, Staginnus U, et al. Pegaspargase in the treatment of Acute Lymphoblastic Leukemia: A CMA model demonstrates its economic benefit for the Italian National Health System. [abstract no. PSY87]. Value Health. 2016;19 (7):A590.

38. Lecureuil C, Staginnus U, Robbins S, et al. Pegaspargase versus asparaginase in childhood acutely lymphoblastic leukemia: a cost-minimization analysis in France. [abstract no. PSY84]. Value Health. 2016;19(7):A589-A90.

39. Villoro R, Aisa F, Domenech M, et al. Pharmacoeconomic assessment of pegaspargase versus asparaginase in acute lymphocytic leukemia. [abstract no. PCN169]. Value Health. 2016;19 (7):A739.

40. US National Institutes of Health. ClinicalTrials.gov [identifier NCT01574274]. 2018.http://www.clinicaltrials.gov/. Accessed 9 Apr 2019.

41. US National Institutes of Health. ClinicalTrials.gov [identifier NCT00671034]. 2018.http://www.clinicaltrials.gov/. Accessed 9 Apr 2019. 